PSFC/JA-11-02

\title{
Observations of Core Toroidal Rotation Reversals in Alcator C-Mod Ohmic L-mode Plasmas
}

Rice, J.E.; Duval*, B.P.; Reinke, M.L.; Podpaly, Y.A.; Bortolon*, A.; Churchill, R.M.; Cziegler, I.; Diamond**, P.H.; Dominguez, A.; Ennever, P.C.; Fiore, C.L.; Granetz, R.S.; Greenwald, M.J.; Hubbard, A.E.; Hughes, J.W.; Irby, J.H.; Ma, Y.; Marmar, E.S.; McDermott***, R.M.; Porkolab, M.; Tsujii, N.; Wolfe, S.M.

* CRPP, EPFL, Lausanne, Switzerland

** UCSD, La Jolla, CA, USA

***currently at IPP Garching, Germany

March 2011

\author{
Plasma Science and Fusion Center \\ Massachusetts Institute of Technology \\ Cambridge MA 02139 USA
}

This work was supported by the U.S. Department of Energy, Grant No. DE-FC0299ER54512. Reproduction, translation, publication, use and disposal, in whole or in part, by or for the United States government is permitted. 


\title{
Observations of Core Toroidal Rotation Reversals in Alcator C-Mod Ohmic L-mode Plasmas
}

\author{
J.E. Rice, B.P. Duval ${ }^{\dagger}$, M.L. Reinke, Y.A.Podpaly, \\ A. Bortolon ${ }^{\dagger \ddagger}$, R.M. Churchill, I. Cziegler, P.H. Diamond ${ }^{\sharp}$, A. Dominguez, P.C. Ennever, \\ C.L. Fiore, R.S. Granetz, M.J. Greenwald, A.E. Hubbard, J.W. Hughes, J.H. Irby, Y. Ma, \\ E.S. Marmar, R.M. McDermott ${ }^{b}$, M. Porkolab, N. Tsujii and S.M. Wolfe \\ Plasma Science and Fusion Center, MIT, Cambridge, MA, USA \\ ${ }^{\dagger}$ CRPP, EPFL, Lausanne, Switzerland \\ ${ }^{\ddagger}$ currently at UC Irvine, CA, USA \\ ${ }^{\sharp} U C S D$, La Jolla, CA, USA \\ ${ }^{b}$ currently at IPP Garching, Germany
}

\begin{abstract}
Direction reversals of intrinsic toroidal rotation have been observed in Alcator CMod Ohmic L-mode plasmas following modest electron density or toroidal magnetic field ramps. The reversal process occurs in the plasma interior, inside of the $q=3 / 2$ surface. For low density plasmas, the rotation is in the co-current direction, and can reverse to the counter-current direction following an increase in the electron density above a certain threshold. Reversals from the co- to counter-current direction are correlated with a sharp decrease in density fluctuations with $\mathrm{k}_{R} \geq 2 \mathrm{~cm}^{-1}$ and with frequencies above $70 \mathrm{kHz}$. The density at which the rotation reverses increases linearly with plasma current, and decreases with increasing magnetic field. There is a strong correlation between the reversal density and the density at which the global Ohmic L-mode energy confinement changes from the linear to the saturated regime.
\end{abstract}




\section{Introduction}

Until recently, plasma rotation and momentum transport in tokamaks haven't received as much attention as particle and energy transport [1]. Since velocity shear is important for its role in reducing turbulence [2,3] and plasma rotation can suppress deleterious MHD modes [4], it is important to address these topics. Usually rotation is generated externally by neutral beam injection, but since momentum input from beams is expected to be low in ITER and future devices, other methods are under consideration. This includes taking advantage of intrinsic rotation, the self-generated flow which arises in the absence of external momentum input. Intrinsic rotation in $\mathrm{H}$-mode and other enhanced confinement regimes is generally directed co-current and has been found to have a relatively simple global scaling [5], with the Mach number proportional to the plasma pressure. In contrast, the intrinsic rotation in L-mode plasmas has a complicated dependence on plasma parameters [6, 7]. In fact, rotation inversions or reversals have been observed $[6,8,9,7,10]$, where the core intrinsic velocity abruptly switches direction, with negligible effect on other macroscopic plasma parameters. Rotation reversals have been induced with changes in density, plasma current, magnetic field and plasma shape. Since the rotation in L-mode plasmas is related to the H-mode power threshold [6], it's important to understand L-mode rotation in its own right. The purpose of the current study is to shed light on this curious reversal phenomenon. In section II the experimental setup on Alcator C-Mod will be described, followed by presentation of reversal time histories, scalings and profile evolution in sections III and IV. Observations of turbulence changes during reversals will be shown in section V, with discussion and conclusions presented in section VI.

\section{Experimental Setup}

These experiments were performed on the Alcator C-Mod tokamak [11], a compact $(\mathrm{R}=0.67 \mathrm{~m}, \mathrm{a} \sim 0.21 \mathrm{~m})$, high magnetic field $\left(\mathrm{B}_{T} \leq 8 \mathrm{~T}\right)$ device with molybdenum plasma facing components. For the plasma conditions considered here, the electron density was between 0.2 and $2.0 \times 10^{20} / \mathrm{m}^{3}$, the plasma current ranged from 0.4 to 1.4 $\mathrm{MA}$ and the toroidal magnetic field was between 2.7 and $7.0 \mathrm{~T}\left(2.6 \leq \mathrm{q}_{95} \leq 7.2\right)$. Discharges were operated in the lower single null (LSN), upper single null (USN) and inner wall limited magnetic field configurations, without auxilliary heating. Plasma elongation ranged from 1.53-1.69, upper triangularity was between 0.22 and 0.33 for LSN and from 0.60 to 0.75 for USN, and lower triangularity ran ges were $0.36-0.50$ for LSN and $0.28-0.32$ for USN. All discharges reported here were in the Ohmic L-mode confinement regime.

Central rotation velocities were measured using a tangentially viewing von Hamos type $\mathrm{x}$-ray spectrometer [12] and complete core rotation profiles were obtained utilizing an imaging $\mathrm{x}$-ray spectrometer system [13]. For the imaging system, there is no external absolute wavelength calibration; calibration was achieved by operating with locked modes which brake the core rotation [14]. If the rotation profile is not stagnant across the entire profile $\left(\mathrm{V}_{T o r}(\mathrm{r})=0\right)$ during a locked mode, then the details of the shapes of the velocity profiles presented in section IV may be different. Edge rotation velocity 
profiles were obtained with a charge exchange recombination spectroscopy (CXRS) system $[15,16]$ using a diagnostic neutral beam (DNB). Electron density and temperature profiles were measured from Thomson scattering [15]. Density fluctuations were observed with phase contrast imaging (PCI) and reflectometer systems [15].

\section{Rotation Reversal Time Histories and Scalings}

The complicated dependence of core intrinsic toroidal rotation on electron density in Ohmic L-mode plasmas has been previously demonstrated for specific discharge conditions [6, 7]: 5.4 T, 0.8 MA, LSN and USN. This parameter set has been expanded to other plasma currents and magnetic fields in the current study. Shown for comparison in Fig. 1 is the scaling of the central toroidal rotation velocity as a function of electron density for a series of individual 5.2 T, lower single null (LSN) Ohmic discharges

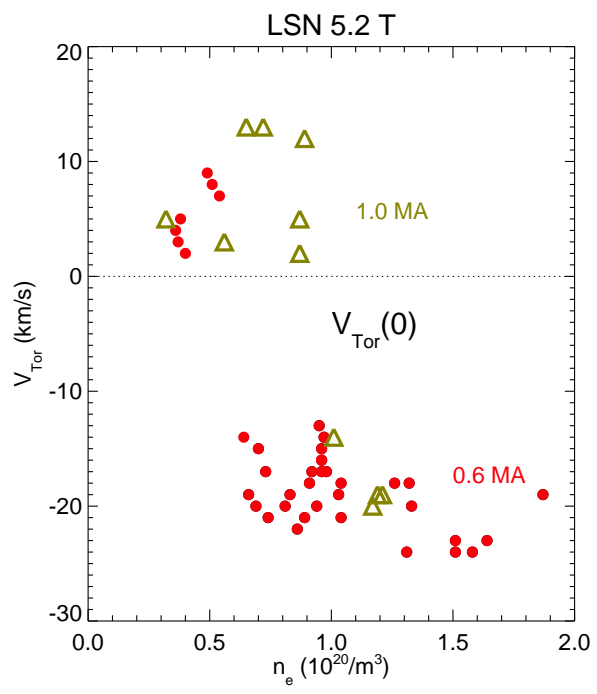

Figure 1: The central rotation velocity as a function of average electron density for LSN, 5.2 T Ohmic discharges with plasma currents of 0.6 MA (dots) and 1.0 MA (triangles).

at plasma currents of 0.6 and 1.0 MA. For the 0.6 MA cases, there is very little dependence on electron density above $0.6 \times 10^{20} / \mathrm{m}^{3}$, and the rotation is in the counter-current direction, with values around $-20 \mathrm{~km} / \mathrm{s}$. As the density is lowered below $0.6 \times 10^{20} / \mathrm{m}^{3}$, the rotation abruptly changes to the co-current direction. Similar behavior is observed at plasma currents of 1.0 MA, but the density at which the rotation reversal occurs is higher, around $1 \times 10^{20} / \mathrm{m}^{3}$. This reversal of the toroidal rotation direction can be observed dynamically during the course of a single discharge by utilizing density ramps $[8,7]$. Shown in Fig.2 are the time histories of three LSN, 5.4 T, 1.05 MA Ohmic 
L-mode discharges ( $\left.\mathrm{q}_{95} \sim 3.2\right)$ with different upward density ramps. In all three cases,

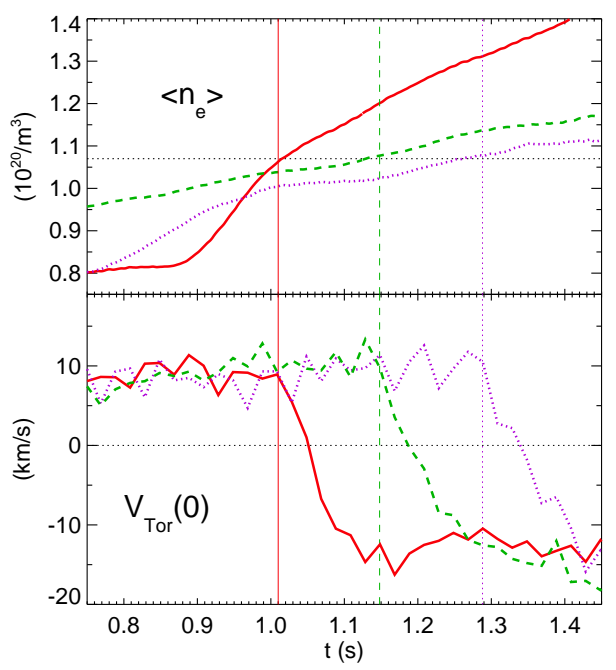

Figure 2: Time histories of the average electron density (top) and central toroidal rotation velocity (bottom) for LSN 1.05 MA 5.4 T $\left(\mathrm{q}_{95}=3.2\right)$ discharges with different density ramps. The dotted horizontal line in the top frame represents an average density of $1.07 \times 10^{20} / \mathrm{m}^{3}$. Vertical lines indicate the initiation times of the three reversals.

the central toroidal rotation velocity changed from $\sim+10 \mathrm{~km} / \mathrm{s}$ (co-current) to $\sim-15$ $\mathrm{km} / \mathrm{s}$ (counter-current) as the electron density passed through $\sim 1.07 \times 10^{20} / \mathrm{m}^{3}$. This rotation reversal usually occurs on a time scale comparable to the L-mode momentum confinement time ( $25 \mathrm{~ms})[17,14]$, although some reversals develop more slowly. The initiation of rotation reversals is quite sensitive to the value of the electron density, as is demonstrated in Fig.3. This plasma experienced two reversals, first from counterto co-current begining at $0.666 \mathrm{~s}$, with a slight decrease in the electron density, and from co- to counter-current begining at $1.146 \mathrm{~s}$, with a slight density increase. Note that the scale for the density trace is greatly expanded. The overall cycle occured with a $10 \%$ change in the electron density. The hysteresis of the process is exhibited in Fig.4, which shows the discharge trajectory in the $\mathrm{n}_{e}-\mathrm{V}_{\text {Tor }}$ plane. The magnitude of the rotation excursion was $\sim 30 \mathrm{~km} / \mathrm{s}$. Whether rotation reversal occurs for a given density ramp depends upon the value of the rotational transform q. Shown in Fig.5 is a comparison of the time histories of three 5.4 T LSN discharges with similar density ramps, but with different plasma currents. Only the discharge at 1.06 MA underwent a rotation reversal for this particular range of densities, from 0.9 to $1.2 \times 10^{20} / \mathrm{m}^{3}$. For the lower plasma currents, the density was above the 'threshold' for reversal from the co- to counter-current direction.

Rotation reversals can also be induced by ramping the plasma current or magnetic field, at fixed electron density. The latter effect is demonstrated in Fig.6 where a com- 


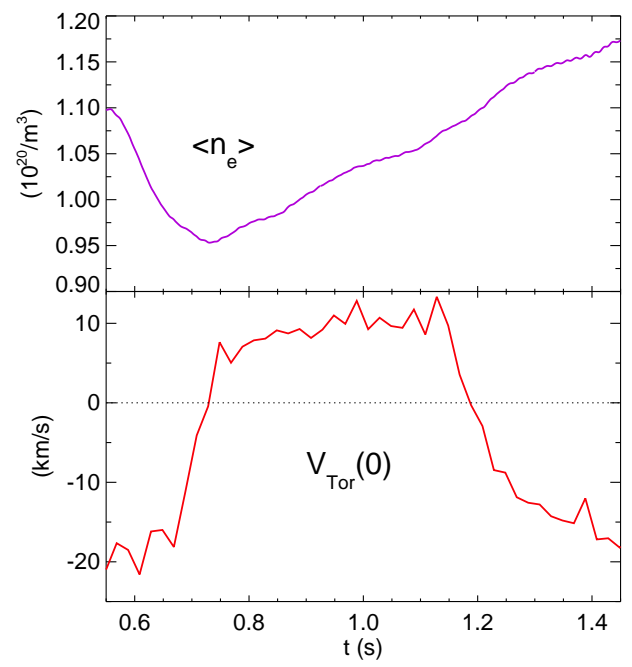

Figure 3: Time histories of the average electron density (top) and central toroidal rotation velocity (bottom) for a LSN 1.05 MA $5.4 \mathrm{~T}\left(\mathrm{q}_{95}=3.2\right)$ discharge with two reversals.

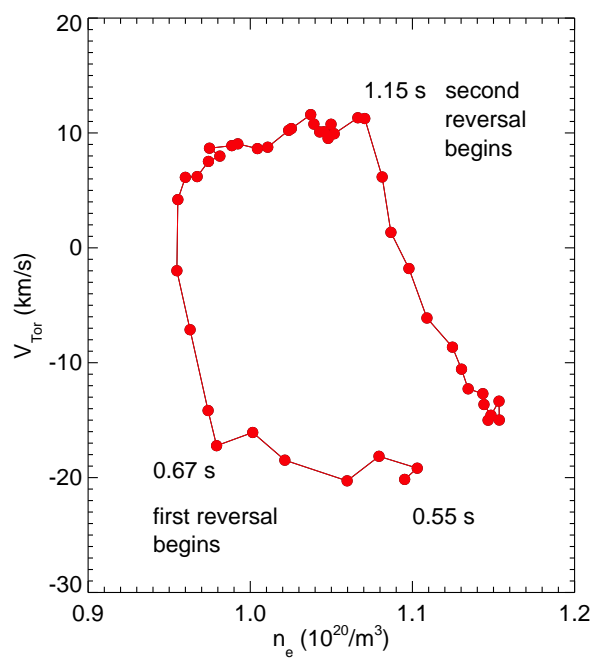

Figure 4: The discharge trajectory in the $\mathrm{n}_{e}-\mathrm{V}_{\text {Tor }}$ plane for the plasma of Fig.3. Points are separated by $20 \mathrm{~ms}$. 


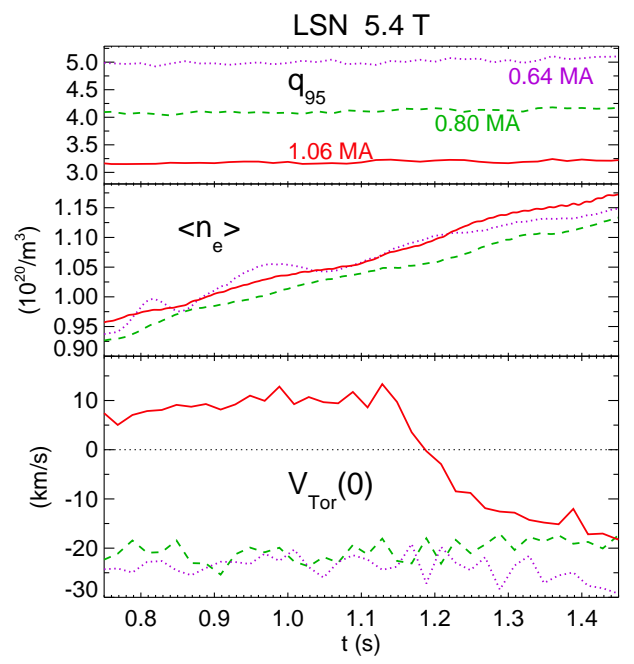

Figure 5: Time histories of $\mathrm{q}_{95}$ (top frame), average electron density (middle frame) and central toroidal rotation velocity (bottom frame) for 5.4 T LSN discharges with plasma currents of $0.64 \mathrm{MA}$ (dotted), $0.80 \mathrm{MA}$ (dashed) and 1.06 MA (solid).

parison is shown of the time histories of two LSN discharges at 0.8 MA and fixed density, one of which had a ramp down and then up of the magnetic field. The discharge with the magnetic field ramp, from $4.5 \mathrm{~T}$ to $3.0 \mathrm{~T}$ and back to $4.5 \mathrm{~T}$, at a relatively constant density, experienced a rotation reversal during the ramp down, and a return to the original rotation velocity after the upward ramp. The discharge trajectory in the $\mathrm{q}_{95^{-}}$ $\mathrm{V}_{T o r}$ plane for the plasma with the magnetic field ramp (solid line in Fig.6) is shown in Fig.7. In this case there is very little hysteresis since the reversals initiated at $\mathrm{q}_{95} \sim 3.0$ for both downward and upward magnetic field ramps. The overall magnitude of the velocity cycle was $\sim 35 \mathrm{~km} / \mathrm{s}$. The reversal density depends both on the magnetic field and plasma current independently. Shown in Fig.8 is a comparison of the time histories of two 0.8 MA upper single null (USN) discharges, with similar downward density ramps, at magnetic fields of 5.4 and $4.1 \mathrm{~T}$. In these cases the rotation changed from counter-current to co-current as the density was ramped down. The reversal for the higher magnetic field plasma occured later in time, at a lower density. A related comparison is presented in Fig.9, for 4.1 T USN discharges at plasma currents of 0.8 and 0.6 MA. For the lower current discharge, the reversal occured later in time, at a lower density; this is consistent with the trend shown in Fig.1.

A large body of rotation reversal data is summarized in Fig.10 where the electron density at the time when the central rotation began to reverse is plotted as a function of plasma current, sorted for different magnetic fields. For 5.4 T discharges, there is a linear increase of the reversal density with plasma current over a factor of three. These points are a mix of LSN, USN and limited discharges; magnetic configuration does 


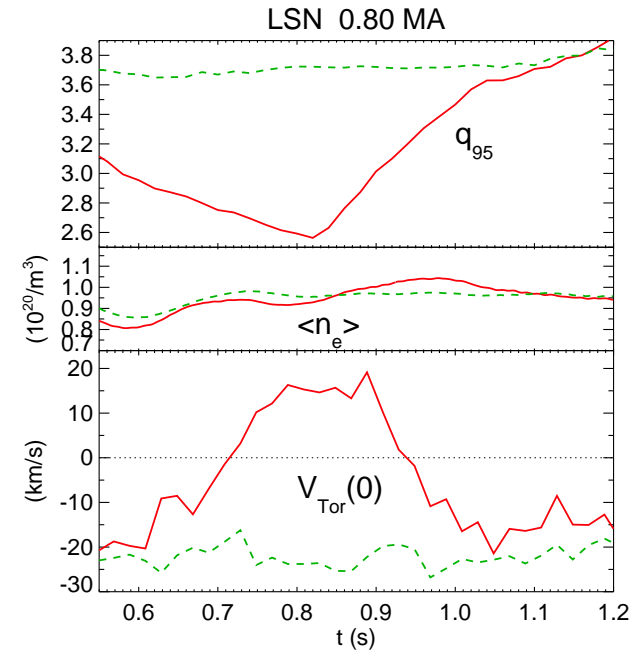

Figure 6: Time histories of 0.8 MA LSN discharges with (solid) and without (dashed) a magnetic field ramp. Same layout as in Fig.5.

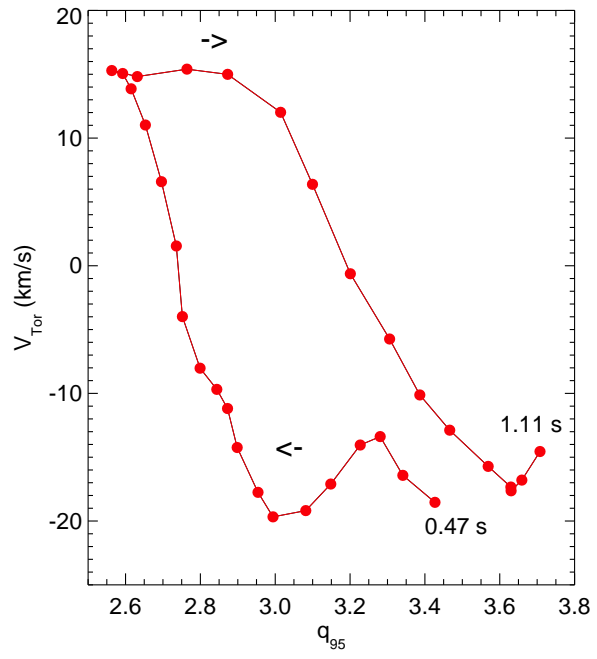

Figure 7: The discharge trajectory in the $\mathrm{q}_{95}-\mathrm{V}_{\text {Tor }}$ plane for the plasma shown by the solid lines in Fig.6. Points are separated by $20 \mathrm{~ms}$. Arrows indicate temporal direction. 


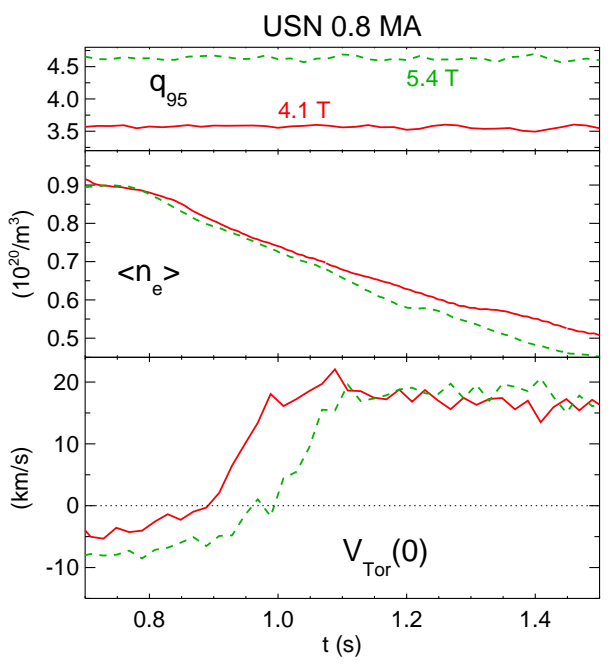

Figure 8: Time histories of 0.8 MA USN discharges at $5.4 \mathrm{~T}$ (dashed) and $4.1 \mathrm{~T}$ (solid).

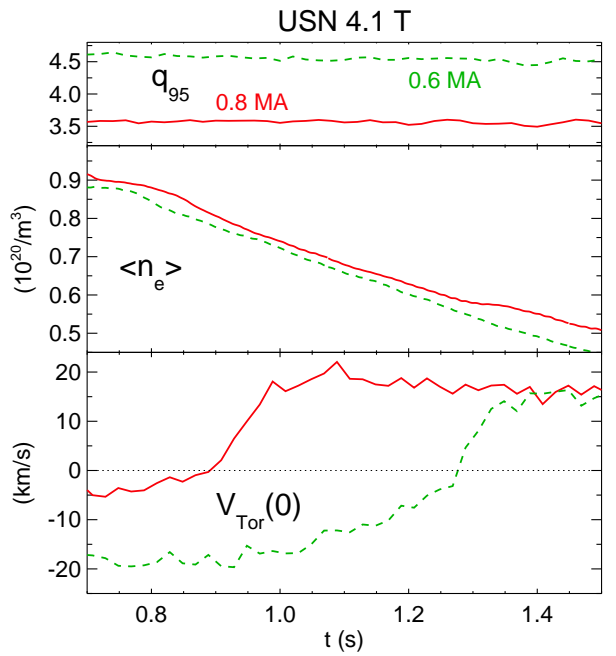

Figure 9: Time histories for two 4.1 T USN discharges, at 0.8 MA (solid) and 0.6 MA (dashed). 


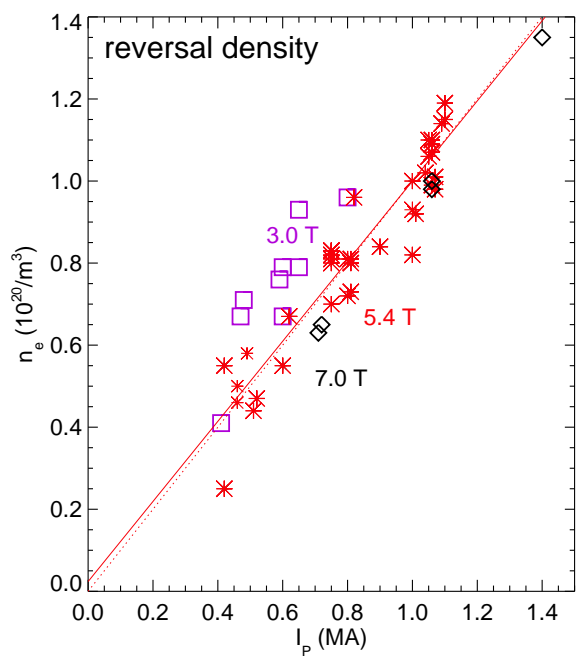

Figure 10: The density at the rotation reversal initiation time as a function of plasma current for $5.4 \mathrm{~T}$ (asterisks), 3.0 $\mathrm{T}$ (squares) and 7.0 $\mathrm{T}$ (diamonds) discharges. The solid line is the best fit to the $5.4 \mathrm{~T}$ data, while the dotted line has a slope of unity.

not seem to affect the reversal density. Some of the scatter is because plasmas with both upward and downward density ramps have been included, and as has been seen in Fig.4, there is a hysteresis of about $10 \%$ in density. Included in the $5.4 \mathrm{~T}$ data set are discharges with reversed magnetic field and plasma current (shown by the smaller asterisks). These reversals occur at electron densities indistinguishable from those with normally oriented current and field. The sense of the reversals is the same, switching from the co-current direction at lower density to counter-current at higher density. Also included on the plot is the best fit line for the $5.4 \mathrm{~T}$ points (solid) and a nearly identical dotted line which has a slope of unity and passes through the origin. A similar trend is apparent for 3.0 T plasmas, although there is an offset, since for the same plasma current, lower magnetic field discharges have a slightly higher reversal density. Similarly, the $7.0 \mathrm{~T}$ points are slightly below the $5.4 \mathrm{~T}$ values. This suggests that $\mathrm{q}_{95}$ may be a better scaling parameter. Shown in Fig. 11 is the density at the start of the reversal as a function of the inverse of the rotational transform q, for different toroidal magnetic fields. For diverted discharges, $1 / \mathrm{q}_{95}$ has been used, while for limited plasmas, $1 / \mathrm{q}(\mathrm{a})$ has been taken. There is a distinct increase of the reversal density with increasing $1 / \mathrm{q}$, although since the lower field points are slightly lower, and the higher field points are slightly higher than the $5.4 \mathrm{~T}$ values, it indicates that the inverse magnetic field dependence is weaker than the plasma current dependence. This point is emphasized in Fig.12, a plot of the reversal density as a function of toroidal magnetic field at fixed plasma current. The best fit curve is proportional to $B^{-0.6}$, weaker than the $1 / B$ dependence of 1/q. The best expression for the relationship between the reversal density, 


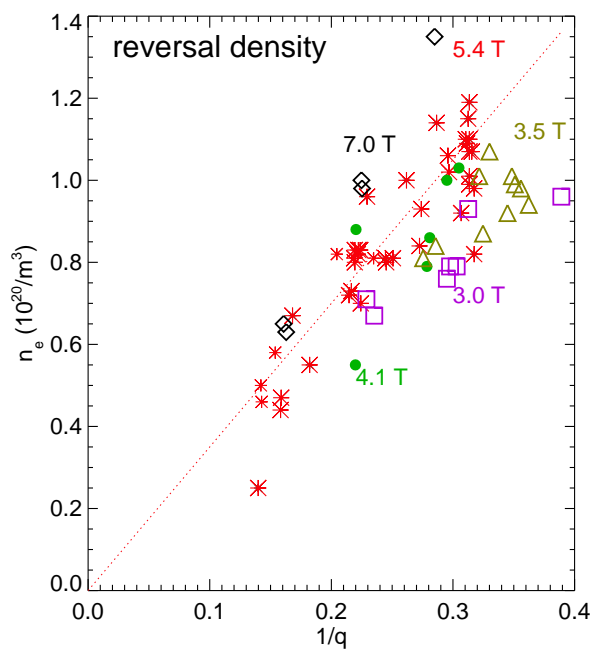

Figure 11: The density at the start of the rotation reversal as a function of $1 / \mathrm{q}\left(1 / \mathrm{q}_{95}\right.$ for diverted discharges) for 7.0 $\mathrm{T}$ (diamonds), 5.4 $\mathrm{T}$ (asterisks), $4.1 \mathrm{~T}$ (dots), $3.5 \mathrm{~T}$ (triangles) and 3.0 T (squares) discharges. The dotted line represents $3.5 / \mathrm{q}$, which is a good approximation to the $5.4 \mathrm{~T}$ points.

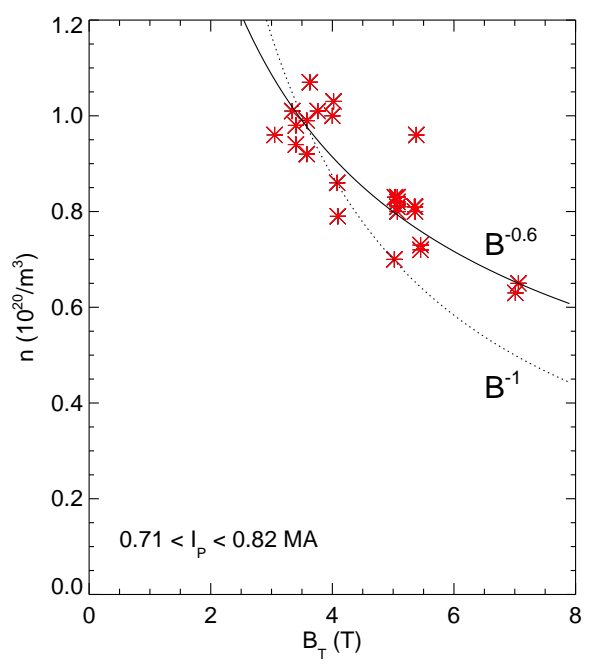

Figure 12: The reversal density as a function of magnetic field, for plasma currents between 0.71 and $0.82 \mathrm{MA}$. The solid curve is proportional to $\mathrm{B}^{-0.6}$ and the dotted curve is proportional to $1 / \mathrm{B}$. 
plasma current and magnetic field can be written $\mathrm{n}_{e} \mathrm{~B}^{0.6} / \mathrm{I}_{p}=2.8$ with $\mathrm{n}_{e}$ in $10^{20} / \mathrm{m}^{3}$, B in $\mathrm{T}$ and $\mathrm{I}_{p}$ in MA. A simple approximation for the $5.4 \mathrm{~T}$ points is $\mathrm{n}_{e} \mathrm{q}_{95}=3.5$.

\section{Rotation Reversal Profile Evolution}

In the previous section, time histories and parameter scalings of the toroidal rotation at the plasma center were shown; in this section the evolution of the entire rotation velocity profile during reversals will be examined. Shown in Fig.13 are the rotation velocity profiles at different times for the discharge shown by the dotted lines in Fig.2, which underwent the reversal process between 1.3 and $1.4 \mathrm{~s}$. The dashed curve repre-

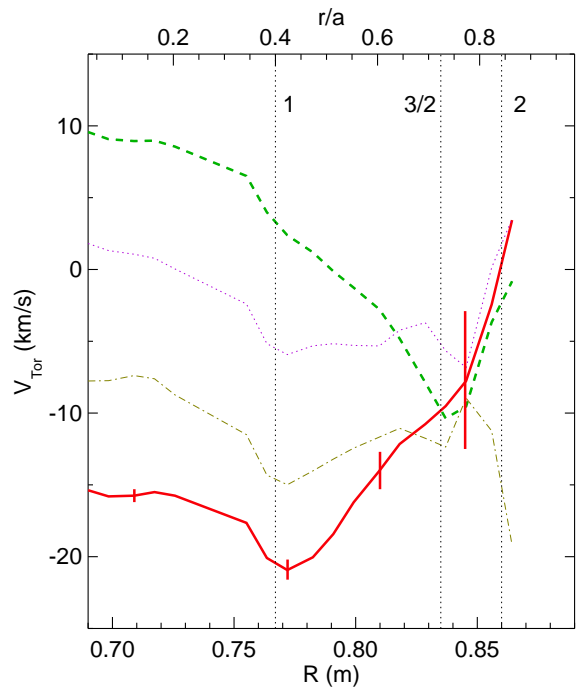

Figure 13: The toroidal rotation velocity profiles at different times for the 1.05 MA, $5.4 \mathrm{~T}\left(\mathrm{q}_{95}=3.23\right)$ LSN discharge shown by the dotted lines in Fig.2. Dashed line, $1.10-1.28 \mathrm{~s}$; dotted line, $1.32-1.34 \mathrm{~s}$; dash-dot line, $1.36-1.38 \mathrm{~s}$; solid line, $1.42-1.60 \mathrm{~s}$. The vertical dotted lines indicate the locations of the $\mathrm{q}=1,3 / 2$ and 2 surfaces. Typical statistical error bars are shown.

sents the rotation velocity profile before $(1.10-1.28 \mathrm{~s})$ the reversal, and is co-current in the core. The solid line indicates the velocity profile after $(1.42-1.60 \mathrm{~s})$ the rotation inversion and is counter-current over most of the profile. Two intermediate profiles are also shown. The reversal occured inside of $\mathrm{R}=0.835 \mathrm{~m}$ ( $\mathrm{r} / \mathrm{a} \sim 0.7$ ), which corresponds to the location of the $q=3 / 2$ surface; the profile was unchanged or anchored outside of this location. The velocity profile inside of the $\mathrm{q}=1$ surface was relatively flat due to sawtooth oscillations, as has been seen on $\operatorname{TCV}[8,9,10]$. The magnetic axis for this discharge was at $\mathrm{R}=0.683 \mathrm{~m}$ and the last closed flux surface (LCFS) was at $\mathrm{R}=$ $0.894 \mathrm{~m}$. The radial electric field at $\mathrm{R}=0.76 \mathrm{~m}$ for this plasma changed from about +5 
$\mathrm{kV} / \mathrm{m}$ before the reversal to around $-15 \mathrm{kV} / \mathrm{m}$ afterwards. Compared to the toroidal rotation velocity profiles, there was very little change in the electron temperature and density profile shapes before and after the reversal, as shown in Fig.14, a situation which has also been documented in TCV plasmas. There was a slight density rise for

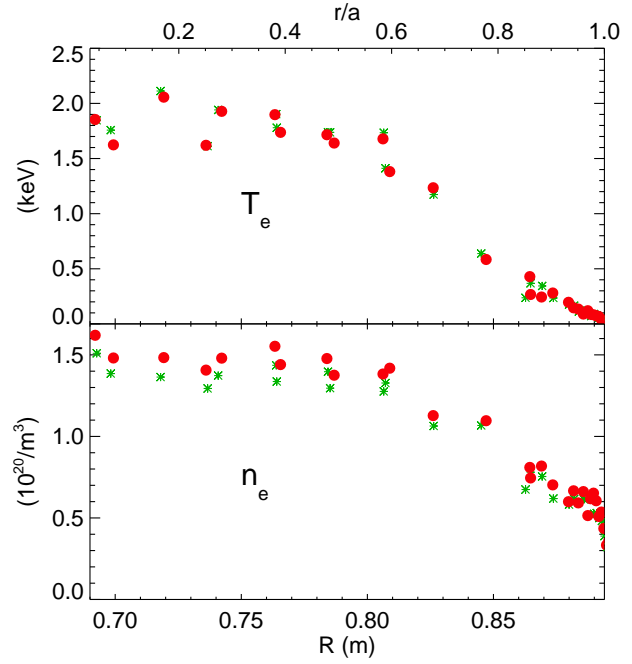

Figure 14: The electron temperature (top frame) and density (bottom frame) profiles from Thomson scattering before (asterisks, $1.15-1.25 \mathrm{~s}$ ) and after (dots, $1.45-1.55 \mathrm{~s}$ ) the rotation reversal for the discharge of Fig.13.

this discharge from 1.1 to $1.6 \mathrm{~s}$ but the overall profile shapes (and gradients, see Fig.27) remained unchanged.

In order to determine if the anchoring position of the rotation velocity profile at $\mathrm{R}=0.835 \mathrm{~m}$ in Fig. 13 during the core reversals is fixed at that location or is tied to the location of the $\mathrm{q}=3 / 2$ surface, discharges with different $\mathrm{q}$ profiles have been investigated. Shown for comparison in Fig. 15 are the velocity profiles at different times for the 0.8 MA, 5.4 $\mathrm{T}\left(\mathrm{q}_{95}=4.67\right)$ USN discharge shown by the dashed lines in Fig.8, which underwent a rotation reversal between 0.9 and $1.1 \mathrm{~s}$. The central rotation was counter-current before the reversal and switched to co-current afterwards. In this case the reversal occured inside of $\mathrm{R}=0.805 \mathrm{~m}$, which for this discharge also corresponds to the location of the $\mathrm{q}=3 / 2$ surface. These profiles were also anchored outside of $\mathrm{q}=$ $3 / 2$ before and after the reversal, and were relatively flat at all times inside of $q=1$. The magnetic axis was at $R=0.676 \mathrm{~m}$ and the LCFS was at $\mathrm{R}=0.888 \mathrm{~m}$. The question of the role of the q profile shape in the reversal process has been addressed by examining the calculated profiles from EFIT [18] reconstructions. A comparison of the inverse magnetic shear gradient scale lengths $\left(\mathrm{R}_{0} / \mathrm{L}_{s} \equiv \hat{\mathrm{s}} / \mathrm{q}\right)$ for the discharges of Figs. 13 and 15 is shown in Fig.16. The profile shapes are a little different near the $q=3 / 2$ surface but the magnitudes at that location are similar, with $\mathrm{R} / \mathrm{L}_{s} \sim 1$. As seen in the top frame, 


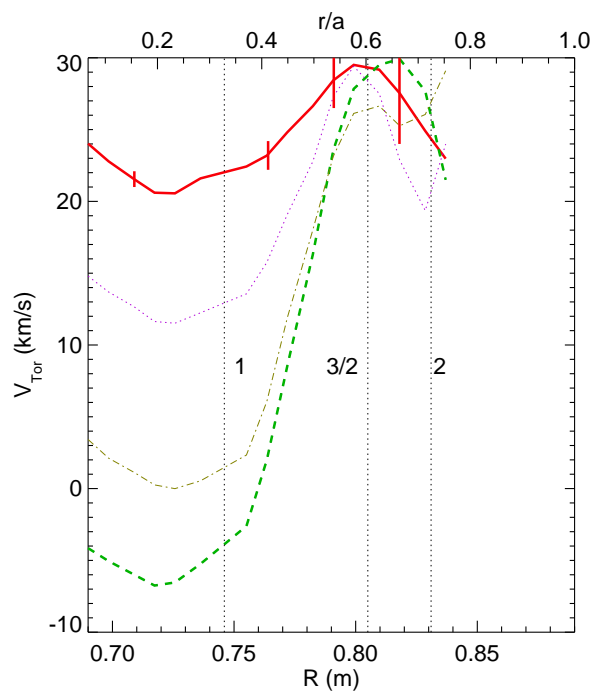

Figure 15: The rotation velocity profiles at different times for the $0.80 \mathrm{MA}, 5.4 \mathrm{~T}$ ( $\mathrm{q}_{95}$ $=4.67$ ) USN discharge shown by the dashed lines in Fig.8. Dashed line, 0.70-0.90 $\mathrm{s}$; dotted line, $0.98-1.00 \mathrm{~s}$; dash-dot line, $1.04-1.06 \mathrm{~s}$; solid line, $1.12-1.32 \mathrm{~s}$. The vertical dotted lines indicate the locations of the $\mathrm{q}=1,3 / 2$ and 2 surfaces.

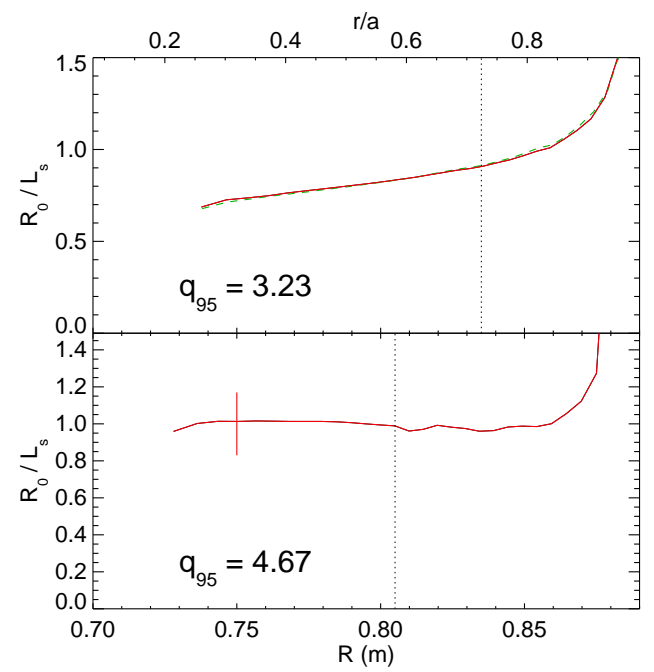

Figure 16: Inverse magnetic shear gradient scale length $\mathrm{R} / \mathrm{L}_{s}$ profiles for the discharges of Fig.13 (top) and Fig.15 (bottom), before (dashed) and after (solid) the rotation reversals. The vertical dotted lines indicate the locations of the $\mathrm{q}=3 / 2$ surfaces. The vertical bar (bottom frame) gives a sense of the uncertainty in the EFIT calculations. 
there was no change in $\mathrm{L}_{s}$ before and after the rotation reversal.

Information on the rotation velocity profile near the plasma edge during the reversal process is available on a very small number of discharges. In Fig. 17 are shown the time histories for four 0.64 MA inner wall limited discharges which all had a magnetic field ramp down to $3.0 \mathrm{~T}$. The main difference between these plasmas was the electron

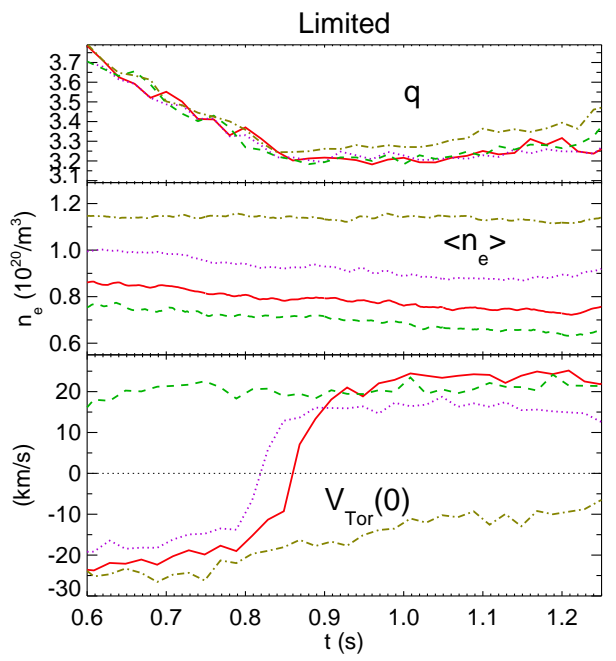

Figure 17: Time history comparison of four 0.64 MA limited discharges.

density. The highest density discharge (dash-dot lines) exhibited counter-current rotation during this time interval while the lowest density plasma (dashed lines) rotated in the co-current direction. The two intermediate density discharges, shown by the solid and dotted lines, experienced rotation reversals from counter- to co-current, with the slight downward density ramps. The core rotation velocity profiles at selected times for these four discharges are shown in Fig.18. The profiles before (dashed lines) and after (solid lines) the reversals are shown in the second and third frames; for the top and bottom frames, there were no inversions. Once again, the reversals occured inside of the $q=3 / 2$ surface and were anchored outside, and the rotation profiles were also relatively flat inside of $q=1$. Rotation velocity profiles outside of $q=2$ are available for the discharge shown by the solid lines in Fig.17. Shown in Fig.19 are the time histories of the toroidal rotation at four locations, $\mathrm{R}=0.690,0.856,0.867$ and 0.881 $\mathrm{m}$, for the plasma shown by the solid lines in Fig.17. The solid line in Fig.19 indicates the core rotation reversal, passing through zero velocity at $t=0.86 \mathrm{~s}$. The velocity time histories near the plasma edge are shown by the symbols. The edge rotation, from the CXRS system, was only available during the diagnostic neutral beam pulses, indicated in the bottom of the figure. At $\mathrm{R}=0.856 \mathrm{~m}$, there was a transient counter-current spike in the rotation as the central velocity passed through zero, but otherwise, the edge rotation was the same before and after the core reversal. There was no change at all 


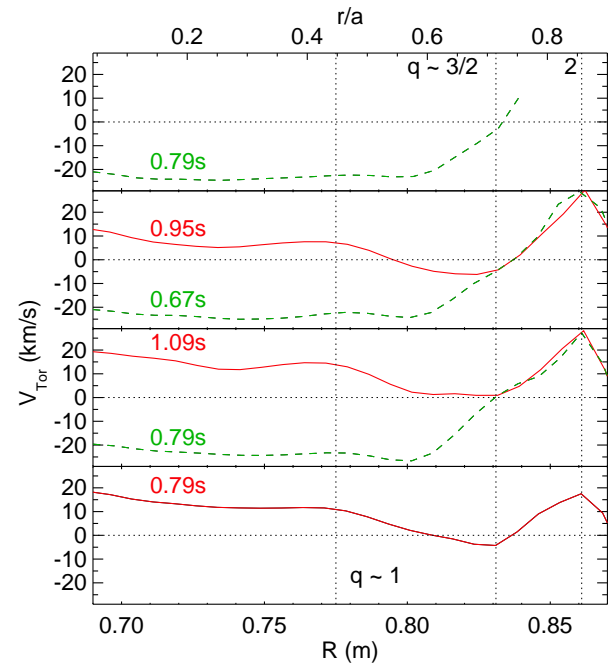

Figure 18: The rotation velocity profiles for the discharges of Fig.17, in order of descending density. Top frame, the dash-dot lines of Fig.17; second frame, the dotted lines of Fig.17; third frame, the solid lines of Fig.17; bottom frame, the dashed lines of Fig.17. The profile times are indicated. Shown by the vertical dotted lines are the locations of the $\mathrm{q}=1,3 / 2$ and 2 surfaces.

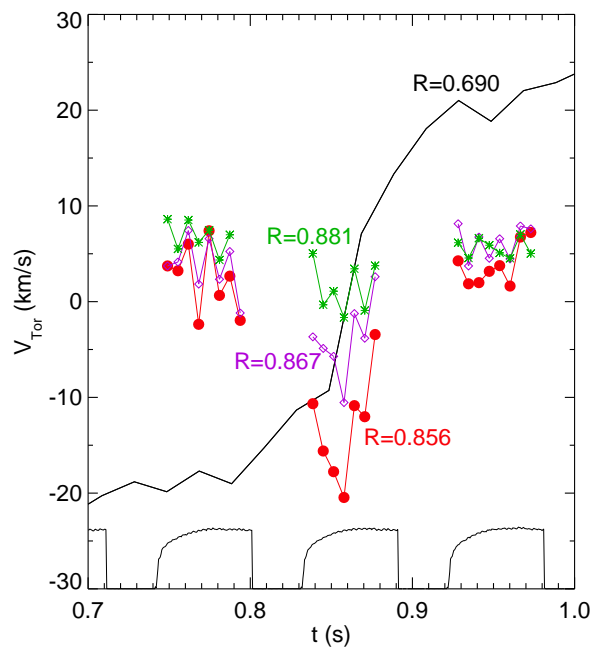

Figure 19: The toridal rotation velocity as a function of time at $\mathrm{R}=0.690$ (solid line), 0.856 (dots), 0.867 (diamonds) and 0.881 (asterisks) $\mathrm{m}$, for the solid line plasma of Fig. 17. At the bottom are shown the diagnostic neutral beam pulses. 
in the velocity at $\mathrm{R}=0.881 \mathrm{~m}$. The edge velocity profiles for this discharge before, during and after the reversal are shown in Fig.20. The edge profiles were flat immedi-

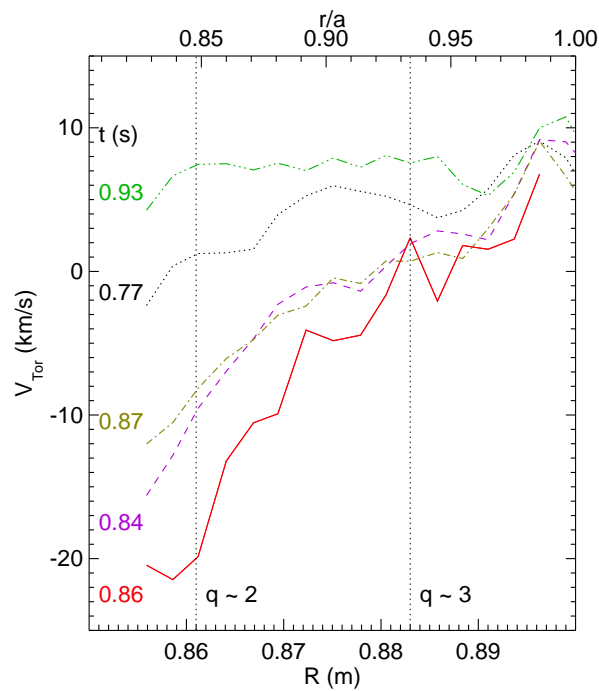

Figure 20: The edge velocity profiles at $\mathrm{t}=0.77$ (dotted line), 0.84 (dashed), 0.86 (solid), 0.87 (dash-dot) and $0.93 \mathrm{~s}$ (dash-dot-dot-dot line). Vertical dotted lines indicate the locations of the $\mathrm{q}=2$ and 3 surfaces.

ately before $(0.77 \mathrm{~s})$ and after $(0.93)$ the reversal, and slightly co-current; these profiles match with the core profiles in the third panel of Fig.18. The maximum of the transient counter-current rotation spike was at $\mathrm{R}=0.86 \mathrm{~m}$, close to the $\mathrm{q}=2$ surface; the profile was anchored outside of $q=3$. The LCFS was at $R=0.90 \mathrm{~m}$. It is not known if this transient rotation spike is universal. This behavior, a pulse of momentum near the $\mathrm{q}=2$ surface in the direction opposite to the final core rotation direction, has been seen on at least one other discharge. Shown in Fig.21 are the rotation velocity time histories at four radial locations, $\mathrm{R}=0.683 \mathrm{~m}$ (the plasma center), $\mathrm{R}=0.757 \mathrm{~m}$ (near $\mathrm{q}=1$ ), $\mathrm{R}=$ $0.830 \mathrm{~m}$ (near $\mathrm{q}=3 / 2$ ) and $\mathrm{R}=0.849 \mathrm{~m}$ (near $\mathrm{q}=2$ ) for the discharge of Fig.13. Inside of $\mathrm{q}=1$, reversal from the co- to counter-current direction is evident. Near $\mathrm{q}=3 / 2$, there was no change during the core reversal. Near the $\mathrm{q}=2$ surface, there was a transient spike in the direction opposite to the final core rotation direction, similar to what was seen in Fig.19. It is possible that this transient rotation pulse plays a role in momentum conservation.

\section{Turbulence Changes During Reversals}

As was shown in Figs.14 and 16, there was very little change in the density, temperature or q profiles (or other macroscopic parameters), while there was a drastic trans- 


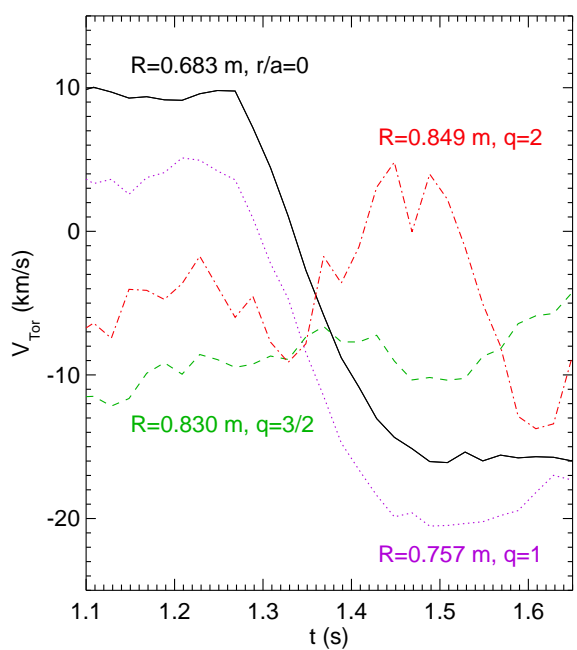

Figure 21: Time histories of the toroidal rotation velocity at $\mathrm{R}=0.683 \mathrm{~m}$ (solid), $\mathrm{R}=$ $0.757 \mathrm{~m}$ (dotted), $\mathrm{R}=0.830 \mathrm{~m}$ (dashed) and $\mathrm{R}=0.849 \mathrm{~m}$ (dash-dot) for the discharge of Fig.13.

formation in the toroidal velocity profiles following the rotation reversals. Significant changes in the character of density fluctuations have also been observed during rotation reversals. Shown in Fig. 22 are dispersion plots of density fluctuations from the PCI diagnostic before and after the core reversal of the discharge shown in Figs. 13 and 2 (dotted lines). There are distinct lobes with $\left|\mathrm{k}_{R}\right| \geq 2 \mathrm{~cm}^{-1}$ for frequencies above $100 \mathrm{kHz}$ which are apparent when the rotation was directed co-current but which disappeared after the reversal. Shown in Fig.23 is the difference between the two spectrograms of Fig. 22 which demonstrate the clear structure of the lobes, which are distinct with $\left|\mathrm{k}_{R}\right|$ $\geq 2 \mathrm{~cm}^{-1}$ for frequencies above $70 \mathrm{kHz}$. The 'slope' of this feature, or apparent phase velocity, is $2 \pi \mathrm{f} / \mathrm{k}_{R} \sim 3 \mathrm{~km} / \mathrm{s}$. This structure extends up to $\mathrm{k}_{R} \sim 10 \mathrm{~cm}^{-1}$ and $\mathrm{k}_{R} \rho_{s}$ is in the range from 0.15 to 0.7 . The feature with positive $\mathrm{k}_{R}$ has a larger intensity than that with negative $\mathrm{k}_{R}$. The direction of propagation is unknown at this time. This feature is not visible at the plasma edge with the gas puff imaging diagnostic. Since the positive and negative slopes in Fig. 23 are the same, this also indicates that this structure exists in the plasma interior where flux surfaces are symmetric.. The time evolution of these features is shown in Fig.24, which depicts the intensity of fluctuations above $180 \mathrm{kHz}$ as a function of time and sorted by $\mathrm{k}_{R}$. For the fluctuations (above $180 \mathrm{kHz}$ ) with $\mathrm{k}_{R} \sim+5 \mathrm{~cm}^{-1}$, there was an abrupt drop in intensity shortly after the core reversal begins. This drop is not due to a Doppler shift out of the observation range. For $\mathrm{k}_{R}$ $\sim-5 \mathrm{~cm}^{-1}$ fluctuations, there was a lingering decay and a slight upshift in wavenumber magnitude. In order to highlight the correlation between the rotation reversal and this structure in the density fluctuations, the evolution of the turbulence intensity for 

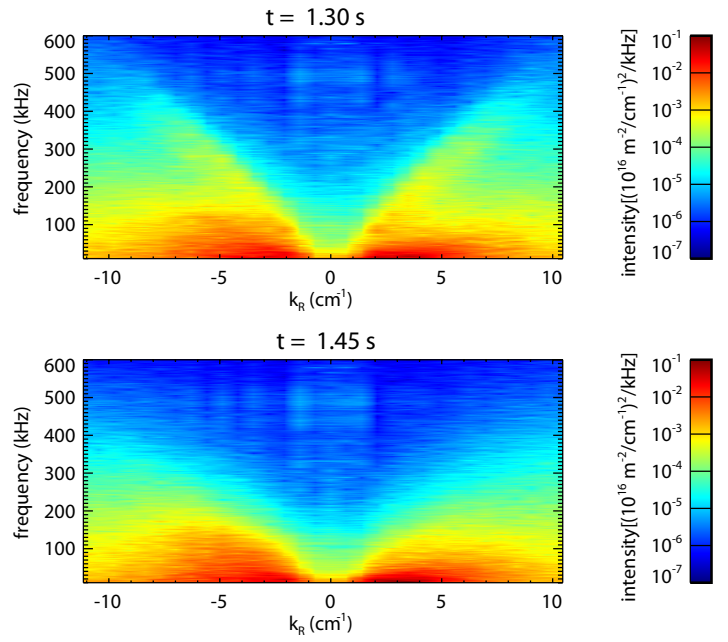

Figure 22: Dispersion plots of turbulence before $(1.30 \mathrm{~s})$ and after $(1.45 \mathrm{~s})$ the rotation reversal in the discharge shown in Fig.13.
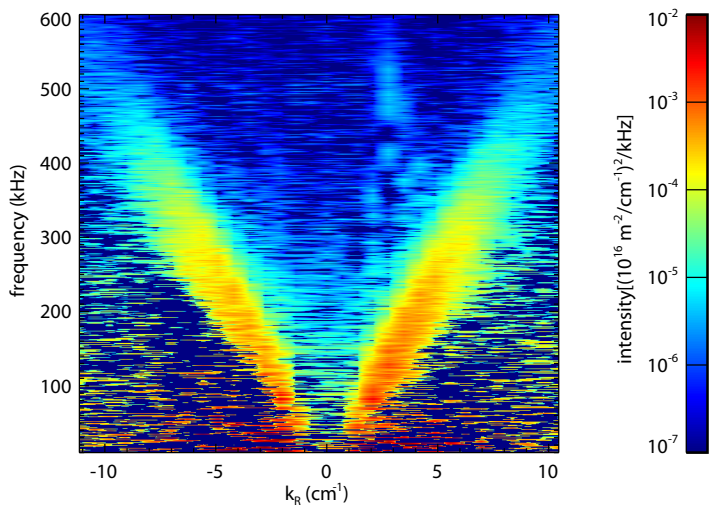

Figure 23: Difference between the two dispersion plots of Fig.22, before (1.30 s) and after $(1.45 \mathrm{~s})$ the rotation reversal. 


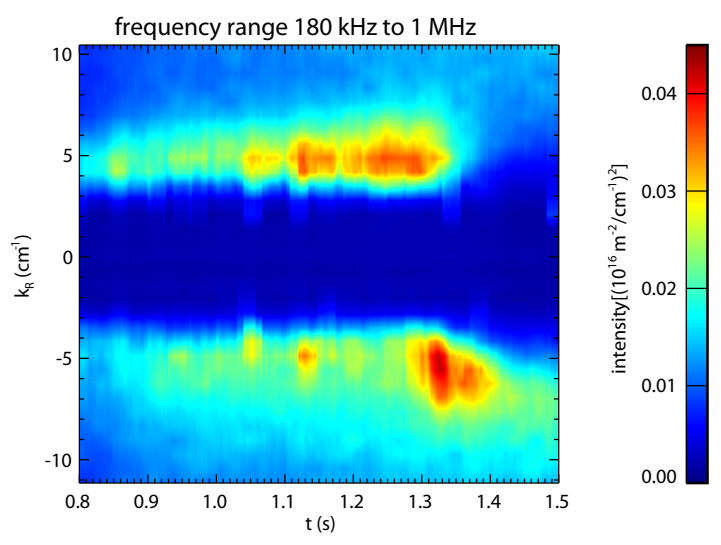

Figure 24: The intensity of density fluctuations above $180 \mathrm{kHz}$, sorted by $\mathrm{k}_{R}$, as a function of time for the discharge of Figs.13 and 22.

the three discharges of Fig.2, with $\mathrm{k}_{R}$ between +4.2 and $+5.6 \mathrm{~cm}^{-1}$ and frequency above $180 \mathrm{kHz}$, is compared to the core velocity time histories in Fig.25. There was an abrupt drop in the fluctuation intensities in these features about $40 \mathrm{~ms}$ after the rotation reversals began. The general rise of the fluctuation levels before the reversals is due to the upward ramps of the electron density in all three discharges, with the strongest ramp shown by the solid curve.

Information on density fluctuations from reflectometry is available on selected discharges. For the $88 \mathrm{GHz}$ channel of this system, the cutoff density is at $0.96 \times 10^{20} / \mathrm{m}^{3}$ and for the $60 \mathrm{GHz}$ channel, the cutoff is at $0.45 \times 10^{20} / \mathrm{m}^{3}$. For a particular $5.07 \mathrm{~T}$ discharge with a plasma current of $0.75 \mathrm{MA}\left(\mathrm{q}_{95}=4.56\right)$, the reversal density (line averaged) was $0.85 \times 10^{20} / \mathrm{m}^{3}$. Since the density profile for this plasma was centrally peaked $\left(\mathrm{n}_{e}(0)=1.35 \times 10^{20} / \mathrm{m}^{3}\right)$, it happens that the cutoff density for the $88 \mathrm{GHz}$ channel was at $\mathrm{R}=0.80 \mathrm{~m}$, just inside of the $\mathrm{q}=3 / 2$ surface, while the $60 \mathrm{GHz}$ channel was viewing the plasma edge. Shown in Fig. 26 is the complex spectrogram time evolution from the $88 \mathrm{GHz}$ channel for this discharge, which underwent the rotation reversal from the co- to counter-current direction begining at $1.11 \mathrm{~s}$. There was a distinct drop in the 'positive' frequency fluctuations from the $88 \mathrm{GHz}$ channel at this time, whereas there was no change in the signal from the outer $60 \mathrm{GHz}$ channel. This supports the observation that the reversal process occurs inside of the $q=3 / 2$ surface. How this manifestation of density fluctuation behavior is related to the mode of Fig. 23 is unknown.

\section{Discussion and Conclusions}




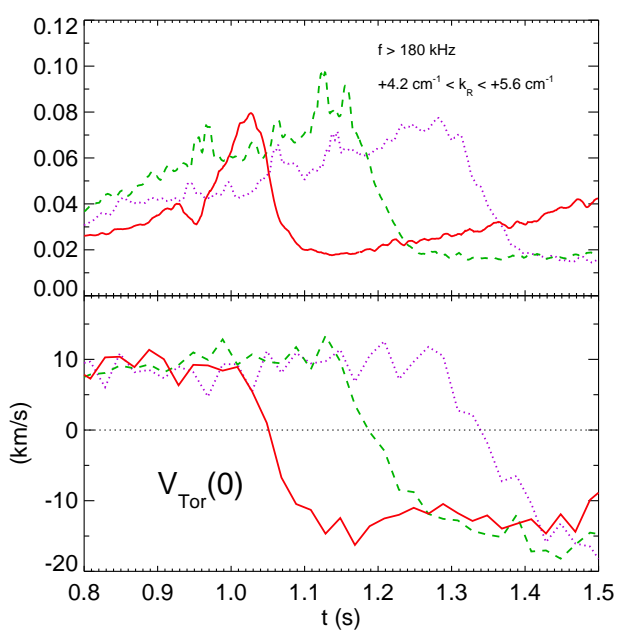

Figure 25: Density fluctuation intensities above $180 \mathrm{kHz}$ and with $+4.2 \mathrm{~cm}^{-1} \leq \mathrm{k}_{R} \leq$ $+5.6 \mathrm{~cm}^{-1}$ (top frame) and core rotation velocities (bottom frame) as a function time for the three discharges of Fig.2.

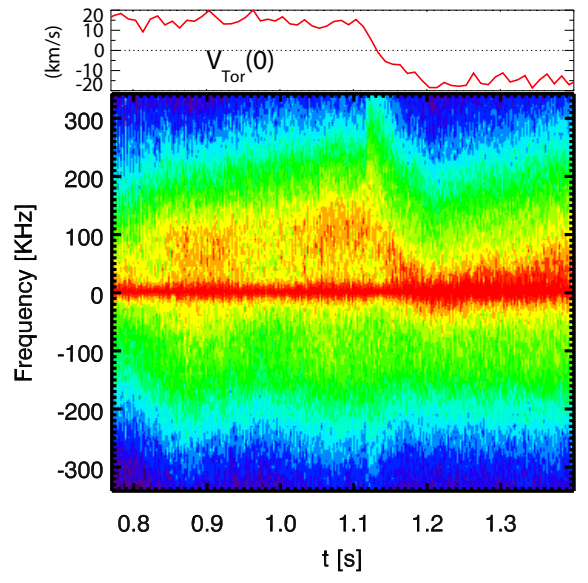

Figure 26: Core rotation velocity (top) and complex spectrogram from the $88 \mathrm{GHz}$ reflectometer channel (bottom) for a $\mathrm{q}_{95}=4.56$ plasma which had a reversal at $1.11 \mathrm{~s}$. 
In order to address the question of what can cause the toroidal rotation to switch direction while other macroscopic parameters remain unchanged, it is informative to examine the momentum flux. The momentum flux can be written as the sum of three terms [19], one proportional to the momentum diffusivity $\chi_{\phi}$, the momentum pinch $\mathrm{V}_{P}$ and the residual stress $\Pi^{r e s}$. A change in sign of any of these could give rise to a change in direction of the toroidal rotation velocity. $\chi_{\phi}$ is not likely directly involved in the reversal process because it is positive definite and cannot change sign. For both ion temperature gradient (ITG) and trapped electron mode (TEM) instabilities, the turbulent equipartition pinch [20] is directed inward and cannot change sign unless the density gradient changes sign. Similarly, the sign of the Coriolis pinch [21] can change only if the density profile gradient changes sign. As shown in Fig.14 (and in Fig.27), the density gradient definitely does not change sign following the rotation reversal. For ITG turbulence, the thermoelectric pinch [20] can be either inward or outward, depending on the mode propagation direction and the proximity to linear marginality. However, this is only expected to play a role for large temperature gradients, such as in an H-mode pedestal, and is probably not important for core L-mode reversals. For TEM turbulence, the thermoelectric pinch can change sign for certain combinations of the values of the temperature and density gradient scale lengths [19]. However, the observed gradient scale lengths do not change before and after the reversals (see Figs.14 and 27), so this is also an unlikely explanation. The temperature and density gradients

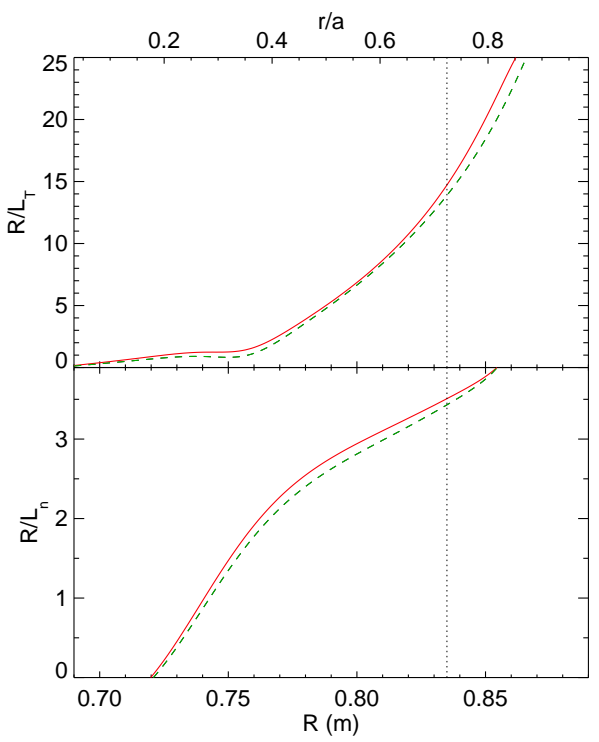

Figure 27: The inverse electron temperature (top) and electron density (bottom) gradient scale lengths before (dashed) and after (solid) the rotation reversal for the discharge of Figs.13 and 14. The vertical dotted line indicates the location of the $q=3 / 2$ surface.

used in Fig.27 were taken from smooth fits to the profiles of Fig.14. It can be concluded that the momentum pinch most likely does not play a role in the reversal process. 
The residual stress can change sign depending on the nature of the underlying turbulence, such as by a change in the mode propagation direction. One example would be a change of wave propagation from the electron diamagnetic drift direction (electron drift waves) to the ion direction (ion drift waves or ITG modes) as the density exceeds a critical threshold [22]. Such a change from TEM (or ETG) to ITG turbulence domination has been invoked to explain the transition in global energy confinement from the linear (neo-Alcator) to saturated Ohmic confinement regimes [23]. In fact, the reversal density of $\sim 0.8 \times 10^{20} / \mathrm{m}^{3}$ for $5.4 \mathrm{~T}, 0.8 \mathrm{MA}$ discharges (Fig.10) is very close to the density separating the linear energy confinement regime from the saturated confinement regime for Ohmic discharges (Fig.1 of [23]). This connection is emphasized in Fig.28 which shows the global energy confinement time (from magnetics) and the core toroidal rotation velocity as a function of electron density for the series of $0.62 \mathrm{MA}$, $5.2 \mathrm{~T}$ discharges shown in Fig.1. The density of the rotation reversal is very close to the

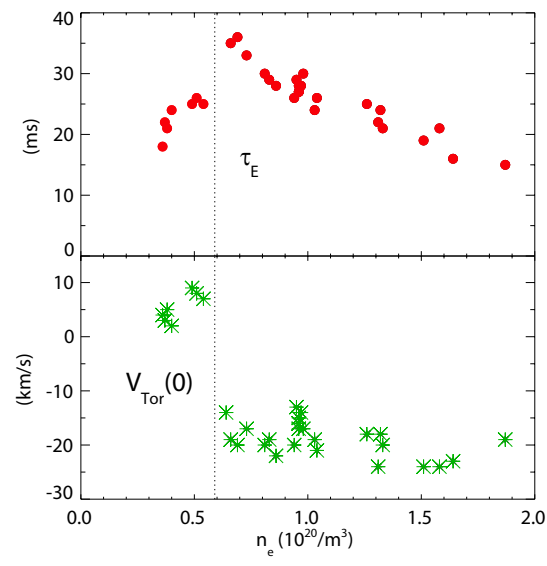

Figure 28: The global energy confinement time (top) and central toroidal rotation velocity (bottom) as a function of average electron density for a series of $0.62 \mathrm{MA}, 5.2 \mathrm{~T}$ $\left(\mathrm{q}_{95}=5.0\right)$ discharges. The vertical dotted line indicates the density which separates coand counter-current rotation.

density which separates the linear increase in global energy confinement from the saturated confinement regime, around $0.6 \times 10^{20} / \mathrm{m}^{3}$ for these conditions. This relationship holds for discharges at different plasma currents as well. A comparison of the rotation reversal density and the transition density from the linear to saturated Ohmic confinement regime, for several different plasma conditions, is shown in Fig.29. There is a very good correlation between these two over a factor of two in density, suggesting a common underlying mechanism. Unfortunately the propagation direction of the feature which is present during the co-current rotation (Fig.23) has not yet been determined. If this propagates in the electron diamagnetic drift direction, then the co-current plasma 


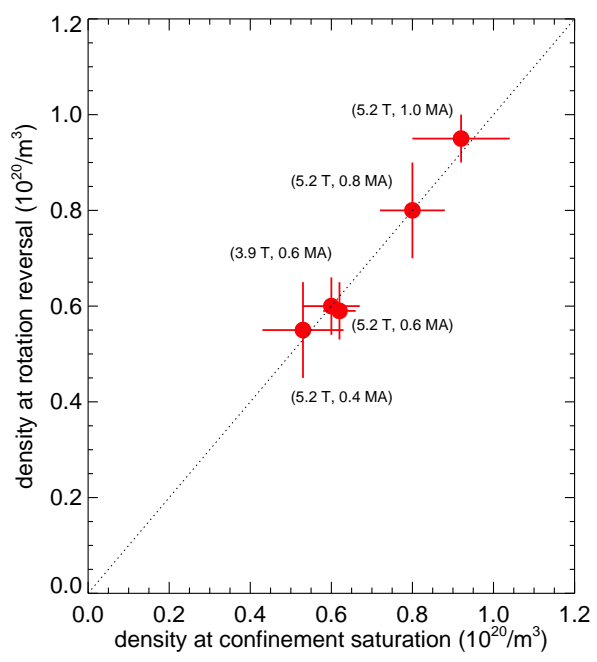

Figure 29: The rotation reversal density as a function of the transition density between linear and saturated energy confinement. Magnetic fields and plasma currents for each point are listed. The dotted line has a slope of unity.

rotation and linear energy confinement would be consistent with CTEM domination of the turbulence at low density.

Other parameters which are known to have an effect on the underlying turbulence, and might be involved in the confinement regime change and rotation reversal process have been considered. For the discharges of Fig.28, there was a monotonic drop in $Z_{e f f}$, the electron and ion temperatures, and the temperature ratio with increasing density, as can be seen in Fig.30. The values of $Z_{e f f}$ and the ratio of $\mathrm{T}_{e} / \mathrm{T}_{i}$ were 2.8 and 1.35, respectively, when the rotation switched from co- to counter-current and the global energy confinement made the transition from the linear to saturated regime. This continuous behavior through the 'transition' density is in contrast to the abrupt changes which occur with the rotation and energy confinement. For 1.0 MA discharges, with a higher reversal density, the values for $\mathrm{T}_{e} / \mathrm{T}_{i}$ and $\mathrm{Z}_{\text {eff }}$ at the reversal and confinement change were somewhat lower, 1.26 and 2.1 , respectively.

The strong correlation between rotation reversals, confinement regime change and turbulence behavior suggests that all three are intimately related. The feature of Fig.23 is only apparent when the rotation is in the co-current direction and the energy confinement is in the linear regime, and then disappears when the rotation switches to the counter-current direction and the energy confinement saturates. The rotation reversal is most likely associated with a change in sign of the residual stress when this structure disappears.

Open questions which need to be addressed include: How is momentum conserved during the reversal process? What is the role of the $q=3 / 2$ surface? Why does the 


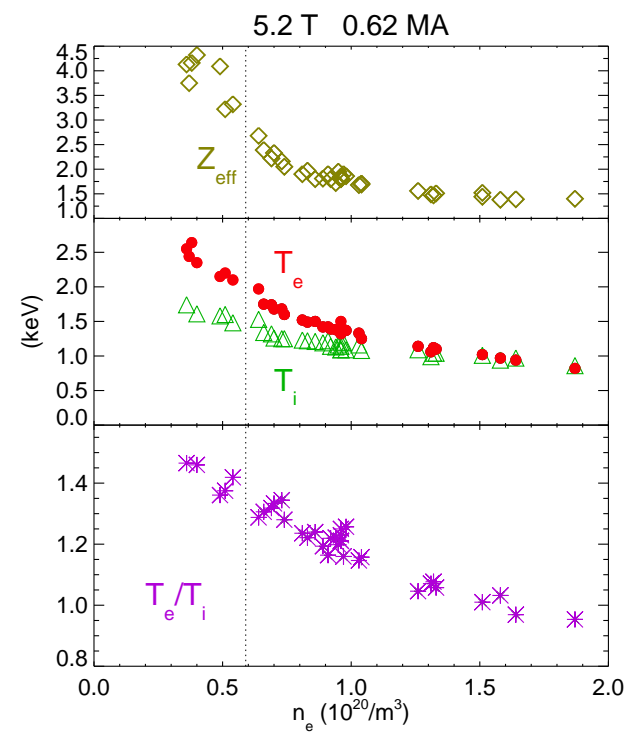

Figure 30: $\mathrm{Z}_{\text {eff }}$ (top), the electron (dots) and ion (triangles) temperatures (middle) and $\mathrm{T}_{e} / \mathrm{T}_{i}$ ratio (bottom) as a function of average electron density for the series of $0.62 \mathrm{MA}$, 5.2 $\mathrm{T}$ discharges shown in Fig.28. The vertical dotted line indicates the density which separates co- and counter-current rotation.

reversal density scale with plasma current? Is there a connection between the reversal density, the density of confinement saturation and the minimum density in the L-H power threshold? What determines the magnitude of the hysteresis in density? What determines the magnitude of the reversal velocity? Regarding the last question, there is some evidence that the magnitude of the reversal is larger for lower toroidal magnetic field plasmas. Shown in Fig.31 is the magnitude of the reversal velocity change as a function of toroidal magnetic field.

In summary, direction reversals of intrinsic toroidal rotation have been observed in Alcator C-Mod Ohmic L-mode plasmas following modest electron density or toroidal magnetic field ramps. The reversal process occurs in the plasma interior, inside of the $\mathrm{q}=3 / 2$ surface. For low density plasmas, the rotation is in the co-current direction, and can reverse to the counter-current direction following an increase in the electron density above a certain threshold. Reversals from the co- to counter-current direction are correlated with a sharp decrease in density fluctuations with $\mathrm{k}_{R} \geq 2 \mathrm{~cm}^{-1}$ and frequencies above $70 \mathrm{kHz}$. The density at which the rotation reverses increases linearly with plasma current, and decreases with increasing magnetic field. There is a strong correlation between the reversal density and the density at which the global Ohmic L-mode energy confinement changes from the linear to the saturated regime.

\section{Acknowledgements}




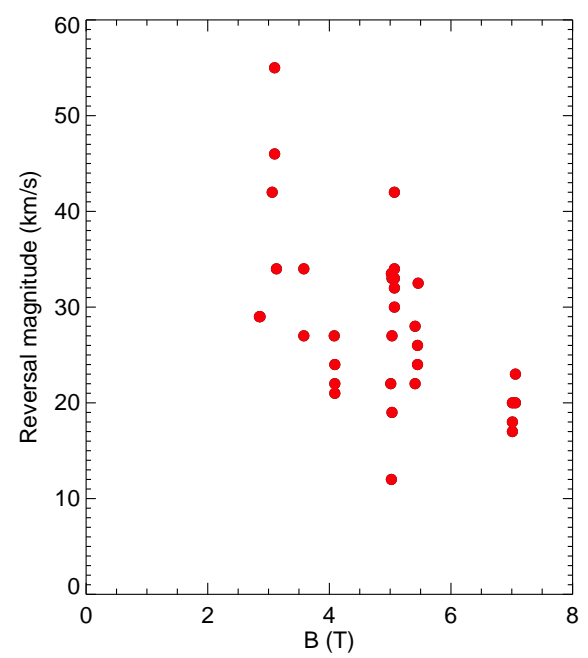

Figure 31: The magnitude of the rotation reversal velocity change as a function of the toroidal magnetic field.

The authors thank J.L. Terry, J. Dorris, D. Ernst, L. Lin and T.S. Hahm for enlightening discussions, and the Alcator C-Mod operations group for expert running of the tokamak. Work supported at MIT by DoE Contract No. DE-FC02-99ER54512.

\section{References}

[1] P.W.Terry, Rev. Mod. Phys. 72 (2000) 109.

[2] T.S.Hahm, Phys. Plasmas 1 (1994) 2940.

[3] K.Burrell, Phys. Plasmas 4 (1997) 1499.

[4] E.J.Strait et al., Phys. Rev. Lett. 74 (1994) 2483.

[5] J.E.Rice et al., Nucl. Fusion 47 (2007) 1618.

[6] J.E.Rice et al., Nucl. Fusion 45 (2005) 251.

[7] J.E.Rice et al., Plasma Phys. Contr. Fusion 50 (2008) 124042.

[8] A.Bortolon et al., Phys. Rev. Lett. 97 (2006) 235003.

[9] B.P.Duval et al., Plasma Phys. Contr. Fusion 49 (2007) B195.

[10] B.P.Duval et al., Phys. Plasmas 15 (2008) 056113.

[11] E.S.Marmar et al., Fusion Sci. Technol. 51 (2007) 261. 
[12] J.E.Rice et al., Nucl. Fusion 37 (1997) 421.

[13] A.Ince-Cushman et al., Rev. Sci. Instrum. 79(2008) 10E302.

[14] J.E.Rice et al., Nucl. Fusion 44 (2004) 379.

[15] N.P.Basse et al., Fusion Sci. Technol. 51 (2007) 476.

[16] R.M.McDermott et al., Phys. Plasmas 16 (2009) 056103.

[17] W.D.Lee et al., Phys. Rev. Lett. 91 (2003) 205003.

[18] L.L.Lao et al., Nucl. Fusion 25 (1985) 1611.

[19] P.H.Diamond et al., Nucl. Fusion 49 (2009) 045002.

[20] T.S.Hahm et al., Phys. Plasmas 14 (2007) 055902.

[21] A.Peeters et al., Phys. Rev. Lett. 98 (2007) 265003.

[22] P.H.Diamond et al., Phys. Plasmas 15 (2008) 012303.

[23] L.Lin et al., Plasma Phys. Contr. Fusion 51 (2009) 065006. 